\title{
Arising Issues In Wireless Sensor Networks: Current Proposals And Future Developments
}

\author{
Saima Maqbool, Ulya Sabeel \\ (Computer Science Department, ASET/Amity University, India)
}

\begin{abstract}
Sensor Networks have been heralded as one of 21 most enabling technologies for 21 st century by Business Week. In recent times wireless sensor networks have received significant attention from researchers due to its unlimited potential. However despite all this effort at providing improved services in these areas, issues still remain unsolved and many more have arised based on the proffered solutions. This paper is focused on presenting to the reader arising issues in wireless sensor networks. We have concentrated on grouping issues in Wireless sensor network into three broad groups namely; the technical issues, the design issues, the system issues, the communication protocol issues, topology issues, key management issues, other wsn issues and recent and research issues. By solving these issues, we can close the wide gap between Wireless Sensor Network Technology invention and its deployment on the field.
\end{abstract}

Keywords - Challenges, Enabling technologies, Issues, Research areas, Wireless sensor networks

\section{INTRODUCTION}

Sensor networks are dense wireless networks of small, low-cost sensors, which collect and disseminate environmental data. Wireless sensor networks facilitate monitoring and controlling of physical environments from remote locations with better accuracy [2]. They have applications in a variety of fields such as environmental monitoring, indoor climate control, surveillance, structural monitoring, medical diagnostics, disaster management, emergency response, ambient air monitoring and gathering sensing information in inhospitable locations $[3,4,5]$.A sensor network is a computer network composed of a large number of sensor nodes. [6] The sensor nodes are densely deployed inside the phenomenon, they deploy random and have cooperative capabilities. Usually these devices are small and inexpensive, so that they can be produced and deployed in large numbers, and so their resources in terms of energy, memory, computational speed and bandwidth are severely constrained. There are different Sensors such as pressure, accelerometer, camera, thermal, microphone, etc. They monitor conditions at different locations, such as temperature, humidity, vehicular movement, lightning condition, pressure, soil makeup, noise Levels, the presence or absence of certain kinds of objects, mechanical stress levels on attached objects, the current characteristics such as speed, direction and size of an object. Normally these Sensor nodes consist there components: sensing, processing and communicating. [7] The various characteristics of wireless sensor networks are Lifetime, Flexibility, Scalability, Responsiveness (and Latency), Maintenance, Data Collection. Even though sensor networks are a special type of ad hoc networks, the protocols designed for ad hoc networks cannot be used as it is for sensor networks due to the following reasons:

1. The number of nodes in sensor networks is very large and has to scale to several orders of magnitude more than the adhoc networks and thus require different and more scalable solutions.

2. The data rate is expected to be very low in WSN and is of statistical in nature. But mobile ad hoc network (MANET) is designed to carry rich multimedia data and is mainly deployed for distributed computing.

3. A sensor network is usually deployed by a single owner but MANET is usually run by several unrelated entities. [8]

4. Sensor networks are data centric i.e. the queries in sensor network are addressed to nodes which have data satisfying some conditions and unique addressing is not possible as they do not have global identifiers. But MANET is node centric, with queries addressed to particular nodes specified by their unique addresses.

5. Sensor nodes are usually deployed once in their life time and those nodes are generally stationary except a few mobile nodes, while nodes in MANET move in an ad hoc manner.

6. Like MANET sensor nodes are also designed for self configuration, but the difference in traffic and energy consumption require separate solutions. In comparison to adhoc networks, sensor nodes have limited power supply and recharge of power is impractical considering the large number of nodes and the environment in which they are deployed. Therefore energy consumption in WSN is an important metric to be considered.

7. Sensor networks are application specific. One can't have a solution that fits for all the problems.

8. Simplicity is the rule in the WSN. Since sensor nodes are small and there is restriction on energy consumption; the communicating and computing software in the nodes should be of less size and computation efficient than the traditional software used for the same purpose.[9] 
The remainder of this paper is organized as follows. We have given the general introduction of the wireless sensor network and sensor node. We have categorised various issues for sensor networks in the next section and a detailed explanation is given for each issue in the proceeding sections. We have also given some idea about recent and research issues. The last section concludes the paper.

\section{ARCHITECTURE OF WIRELESS SENSOR NETWORK AND SENSOR NODE}

The architectural diagram for Wireless Sensor Network and sensor node architecture are given below in fig 1 and fig 2 .

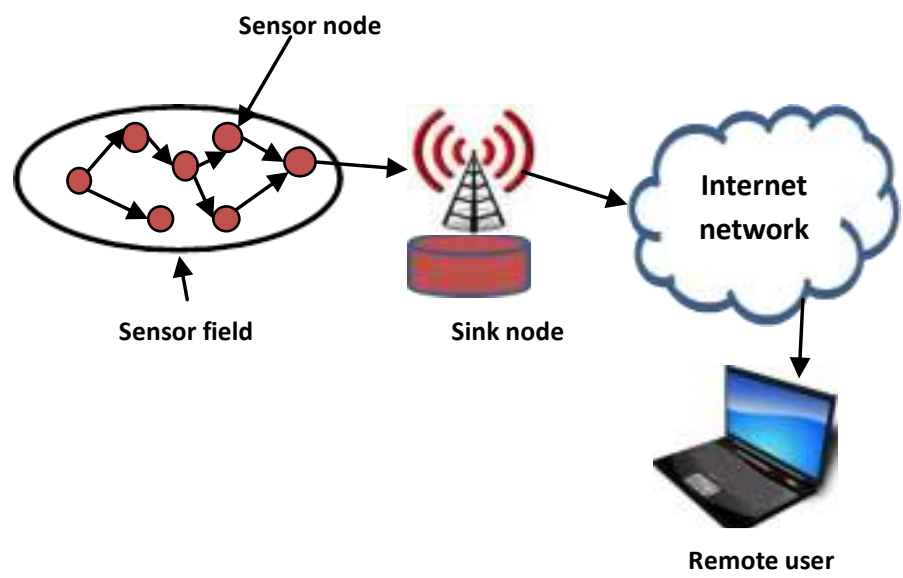

Fig1: Architecture of sensor network
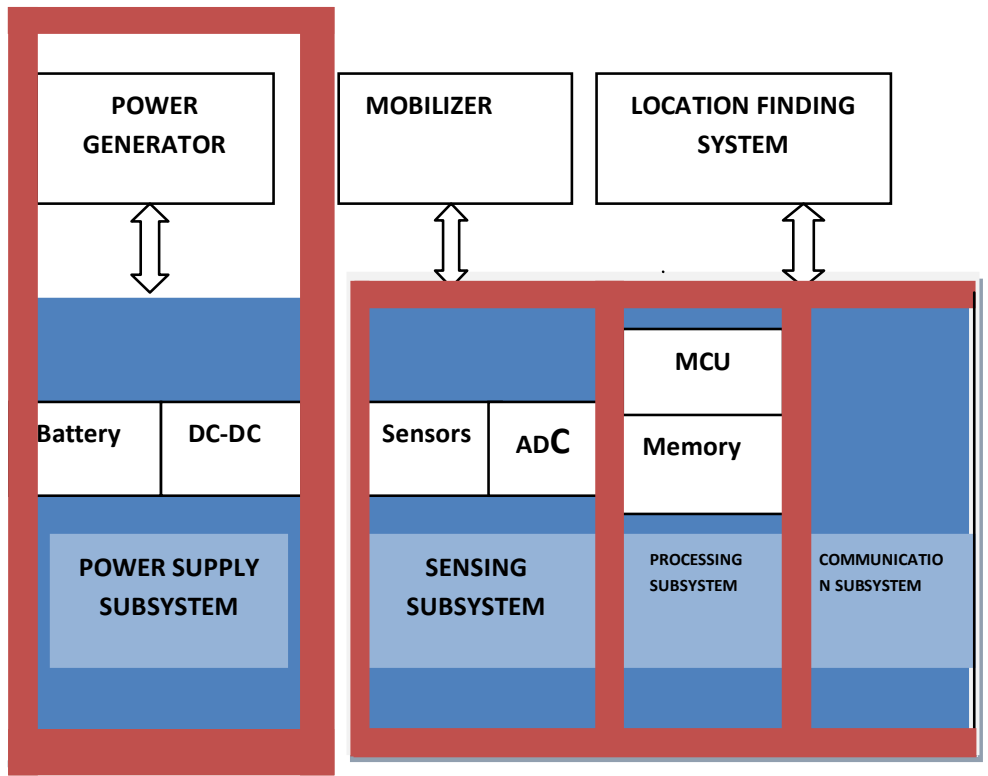

Fig2: Architecture of a sensor

\section{ISSUES IN WIRELESS SENSOR NETWORKS}

In recent years there has been a growing interest in Wireless Sensor Networks (WSN). Recent advancements in the field of sensing, computing and communications have attracted research efforts and huge investments from various quarters in the field of WSN. Also sensing networks will reveal previously unobserved phenomena. The various areas where major research activities going on in the field of WSN are deployment, localization, synchronization, data aggregation, dissemination, database querying, architecture, middleware, security, designing less power consuming devices, abstractions and higher level algorithms for sensor specific issues[9]. 


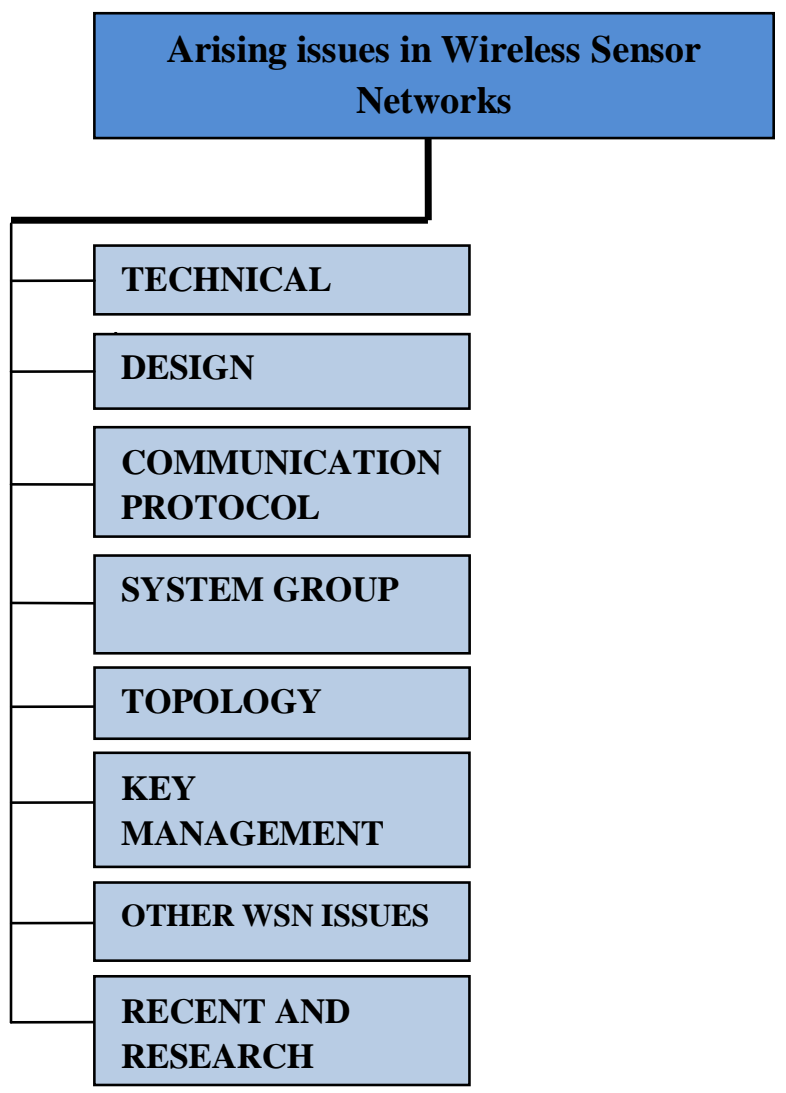

Fig 3: Issues in wsn

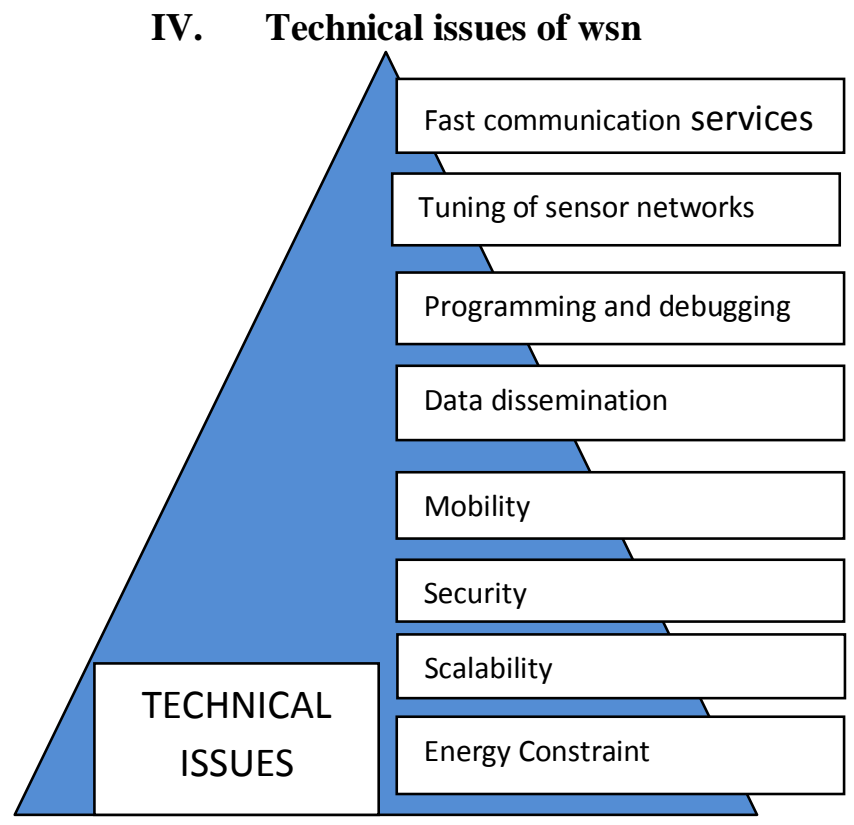

Fig 4: Technical Issues

4.1 Fast communication services: A sensor node expends the maximum energy in data communication as shown in Figure 3.2. Communication is performed by the transceiver circuitry during both receiving and transmitting data. The breakdown of the transceiver power consumption shows that a transceiver expends a similar amount of energy for transmitting and receiving, as well as when it is idle. Moreover, a significant amount of energy can be saved by turning off the 
transceiver to a sleep state whenever the sensor node does not need to transmit or receive any data. This provides energy savings of up to $99.99 \%$ (from $59.1 \mathrm{~m} \mathrm{~W}$ to $3 \mu \mathrm{W}$ ). [10]

4.2 Tuning of sensor networks: Writing software for sensor networks requires full understanding of the physical phenomenon under observation. Nevertheless, in many cases knowing the details of the area of operation is difficult or unfeasible. This leads to the necessity of configuring and tuning the application executed on the sensor nodes even after the network has been deployed and a layer of computation is inserted between the application and the sensing equipment. This layer acts like a tiny interpreter and can be used to customize the behavior of already running applications. [11]

4.3 Programming and debugging: Currently, programmers are too much concerned with low level details like sensing and node to node communication raising a need for programming abstractions. There is considerable research activity for designing programming models for sensor networks due to following issues:

1) Since the data collected from the surrounding phenomenon is not for general purpose computing we need a reactive, event driven programming model.

2) Resources in a sensor network are very scarce, where even a typical embedded OS consuming hundreds of KB of considered too much. So programming models should help programmers in writing energy efficient applications.

3) We need to reduce the run time errors and complexity since the applications in a sensor network need to run for a long duration without human intervention.

4) Programming models should help programmers to write bandwidth efficient programs and should be accompanied by runtime mechanisms that achieve bandwidth efficiency whenever possible.

TinyOS with Nesc and TinyGALS are examples for this category. Improving programming ease in languages such as Nesc and galsC itself provides tremendous opportunities for research. [9]

4.4Data dissemination: Data dissemination is a process by which data and the queries for the data are routed in the sensor network [81]. Data dissemination is a two step process. In the first step, if a node is interested in some events, like temperature or humidity, then it broadcasts its interests to its neighbors periodically and then through the whole sensor network. In the second step, the nodes that have the requested data will send the data back to the source node after receiving the request. . The main difference between data aggregation and data dissemination is, in data dissemination all the nodes including the base station can request for the data while in data aggregation all al the aggregated data is periodically transmitted to the base station. In addition, data aggregation data can be transmitted periodically, while in data dissemination data is always transmitted on demand. Flooding is one important protocol which includes data dissemination approach.

Active Research Areas: Main research focus in data aggregation is geared towards conserving energy. Other research issues include improving security in data transmission and aggregation, handling tradeoffs in data aggregation i.e. tradeoffs between different objectives such as energy consumption, latency and data accuracy, improving quality of service of the data aggregation protocols in terms of bandwidth and end to end delay.[9]

4.5 Mobility: A wireless sensor network (WSN) offers a wide range of applications and it is also an intense area of research. However, current research in wireless sensor networks focuses on WSN where they are deployed in a stationary position providing the base station with information about the subject under observation. However, a mobile sensor network is a collection of WSN nodes. Each of these nodes is capable of sensing, communication and moving around. It is the mobility capabilities that distinguish a mobile sensor network from the conventional 'fixed' WSN. Mobile sensor networks offer many opportunities for research as these sensors involves: the estimate location of the node in a movement scenario, an efficient DATA and information processing schemes that can cope with the mobility measurements and requirements (this includes the routing theory and the potential MAC Protocol Used). [12]

4.6Security: Communications over wireless channels are, by nature, insecure and easily susceptible to various kinds of treats. A large-scale sensor network consists of huge number of sensor nodes and may be dispersed over a wide area. Typical sensor nodes are small with limited communication and computing capabilities. These small sensor nodes are pervious to several key types of treats. [13]

4.7Scalability: While high-density deployment of sensor nodes in a WSN provides redundancy and improves the fault tolerance of the network, this also creates scalability challenges. The number of sensor nodes deployed for sensing a physical phenomenon may be on the order of hundreds or thousands. 
Therefore, the networking protocols developed for these networks should be able to handle these large numbers of nodes efficiently. The density can range from a few to hundreds of sensor nodes in a region, which can be less than $10 \mathrm{~m}$ in diameter. The node density depends on the application for which the sen sor nodes are deployed. [10]

4.8Energy constraint: Energy efficiency is always one of the major goals in the design of WSN. Energy efficient protocols have been explored for a long time. Previous work expects to achieve the goal of energy efficiency by designing energy efficient query protocols [14], routing protocols, such as [15, 16, 17, 18], energy efficient MAC protocols like [15, 19], energy efficient clustering and duty cycle management [20], sensor network topology management, and other energy efficient approaches [16, 17, 18, 21, 22, 19]. However, these approaches mainly focus on finding some energy efficient path, designing better turn on/off schedules, forming energy efficient clusters, and so on, but none of them has examined the energy efficiency from the view of the data itself, i.e., to adapt the data sampling rate to the data dynamics and keep lazy when data consistency is maintained.

\section{Design issues of wsn}

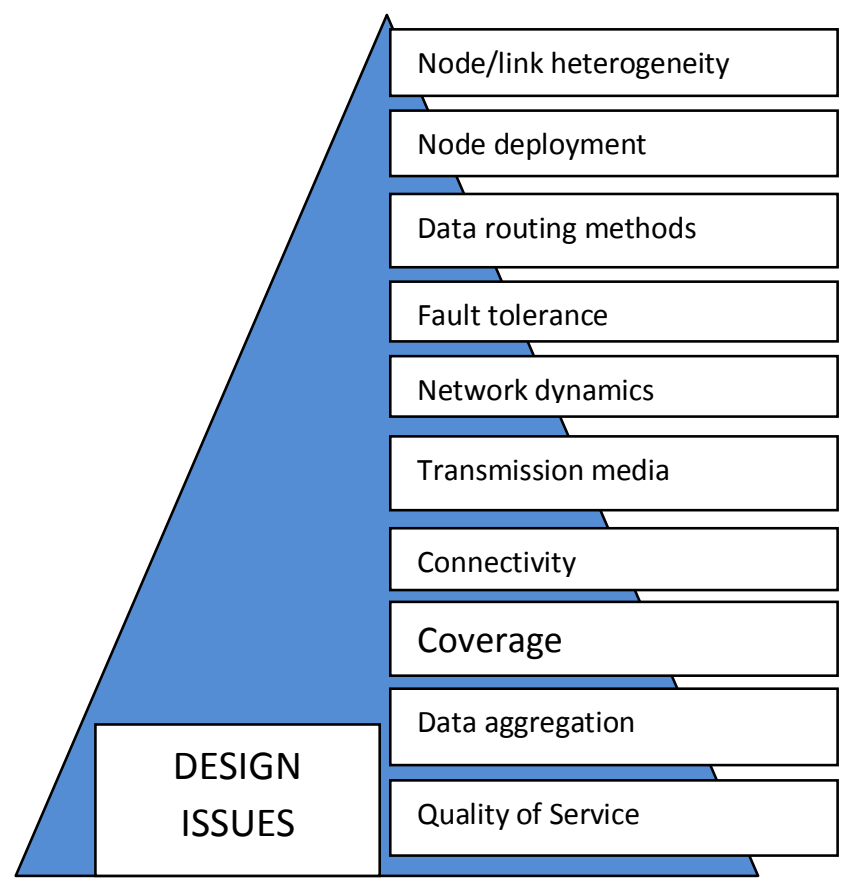

Fig 5: Design Issues

5.1 Node/link heterogeneity: The presence of heterogeneous nodes (i.e., nodes with an enhanced energy capacity or communication capability) in a sensor network is known to increase network reliability and lifetime. However, questions of where, how many, and what types of heterogeneous resources to deploy remain largely unexplored. Energy and link heterogeneity in ad hoc sensor networks and resource-aware MAC and routing protocols are developed to utilize those resources. Properly deployed, heterogeneity can triple the average delivery rate and provide a 5 -fold increase in the lifetime (respectively) of a large batterypowered network of simple sensors. [23]

\subsection{Node deployment:}

Manual deployment: Sensors are manually deployed Data is routed through predetermined path

Random deployment: Optimal clustering is necessary to allow connectivity \& energy-efficiency Multi-hop routing

5.3 Data routing methods: In WSN, the sensor nodes have a limited transmission range, and their processing and storage capabilities as well as their energy resources are also limited. Routing protocols for wireless 
sensor networks are responsible for maintaining the routes in the network and have to ensure reliable multihop communication under these conditions. Various Routing Application-specific methods are:

Time-driven: Periodic monitoring, Event-driven: Respond to sudden changes, Query-driven: Respond to queries, and Hybrid

5.4 Fault tolerance: The hardware constraints lead sensor nodes to frequently fail or be blocked for a certain amount of time. These faults may occur because of a lack of power, physical damage, environmental interference, or software problems. The failure of a node results in disconnection from the network. Since the WSN is interested in information regarding the physical phenomenon instead of information from a single sensor, the failure of a single node should not affect the overall operation of the network. The level of failures that is allowed by the network to adequately continue its functions defines its fault tolerance. [10]

5.5 Network dynamics: Network dynamics may arise from node failures, wireless link failures, node mobility, and node state transitions due to the use of power management or energy efficient schemes. Such a highly dynamic network greatly increases the complexity of QoS support. [24]

5.6 Transmission media: The successful operation of a WSN relies on reliable communication between the nodes in the network. In a multi-hop sensor network, nodes can communicate through a wireless medium creating links between each other. These links can be formed by radio, infrared, optical, acoustic or magneto-inductive links. To enable inter operability and global operation of these networks, the chosen transmission medium must beavailable worldwide. [10]

5.7 Connectivity: Wireless sensor nodes are resource constrained and mostly operate in unsafe environments. Displacement, communication blockage and frequent failure of wireless sensor nodes cause network topology to become very dynamic that affects network connectivity. Lack of topological connectivity between nodes may lead the entire network to break apart into disconnected pieces. The disconnected subnetworks may be unable to communicate with the sink, hence cannot send its data to base station. Many researchers have devoted their efforts to develop network communication schemes that have proven better network connectivity, communication performance and prolonged network lifetime. [25]

5.8 Coverage: Coverage Problem is one of the active issues of the WSNs that determine how efficiently the sensor network is being covered by a set of sensor nodes. This problem deals with the QoS of the network ensuring that the particular sensor network is monitored or observed by at least one sensor node.

It may be broadly classified into three main categories:

- Area Coverage where the sensor nodes are deployed to cover a specific area or region

- Target Coverage Problem where the sensor nodes are deployed to cover a specific set of targets or points.

- Coverage dealing with the determination of the maximum support /breach path. [26]

2.9 Data aggregation: Data Aggregation is defined as the process of aggregating the data from multiple sensors to eliminate redundant transmission and estimating the desired answer about the sensed environment, then providing fused information to the base station. Some design issues in data aggregation are [27, 28]:

1. Sensor networks are inherently unreliable and certain information may be unavailable or expensive to obtain; like the number of nodes present in the network and the number of nodes that are responding and also it is difficult to obtain complete and up-to date information of the neighboring sensor nodes to gather information.

2. Making some of the nodes to transmit the data directly to the base station or to have less transmission of data to the base station to reduce energy.

3. Eliminate transmission of redundant data using meta- data negotiations as in SPIN protocol.

4. Improving clustering techniques for data aggregation to conserve energy of the sensors.

5. Improving In-Network aggregation techniques to improve energy efficiency. In-Network aggregation means sending partially aggregated values rather than raw values, thereby reducing power consumption. [9]

2.10 Quality of service: Quality of Service (QoS) always has very key role for all types of applications in networks, including conventional, wireless ad hoc and wireless sensor network. QoS routing is performed usually through resource reservation in a connection oriented communication in order to meet the QoS requirement for each individual connection. While couple of different mechanisms have been proposed for routing QoS constrained image and video type of data in wire based network, they cannot be directly applied to wireless sensor network, because of its different architecture, structure and resource constraints. Therefore 
several new protocols have been proposed for QoS routing in wireless networks taking the dynamic nature of the network into account. Some of the proposed protocols consider the imprecise state information while determining the routes. [29]

\section{Communication protocol issues}

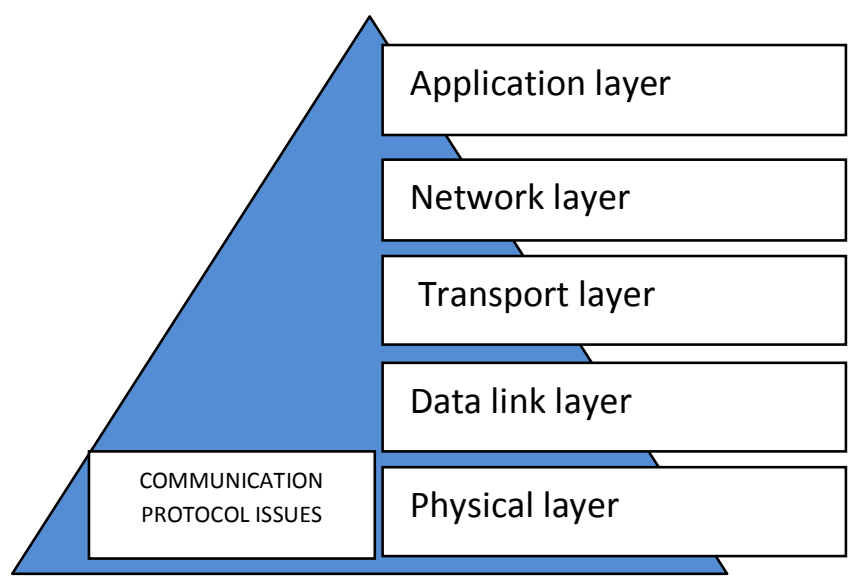

Fig 6: Communication Protocol Issues

A network consist of several nodes, each nodes uses the protocol stack to communication with each other. Hence researchers must focus on developing a reliable and energy efficient protocol stack. It is important to optimize communication and minimize energy usage. However traditional networking protocols do not work well in a WSN since they are not designed to meet WSN requirement. Design issues still remain in each of the five standard protocol layers, this include; application layer, transport layer, network layer, data-link layer, and physical layer.

3.1 Application layer: Different applications can be carried on and used on the application layer depending on the tasks. Responsible for traffic management and provide software for different applications that translate the data in an understandable form or send queries to obtain certain information. Sensor networks deployed in various applications in different fields, for example; military, medical, environment, agriculture fields.

3.2 transport layer issues: End to End reliable communication is provided at Transport layer. The various design issues for Transport layer protocols are [30, 31]:

1. In transport layer the messages are fragmented into several segments at the transmitter and reassembled at the receiver. Therefore a transport protocol should ensure orderly transmission of the fragmented segments.

2. Limited bandwidth results in congestion which impacts normal data exchange and may also lead to packet loss.

3. Bit error rate also results in packet loss and also wastes energy. A transport protocol should be reliable for delivering data to potentially large group of sensors under extreme conditions.

4. End to End communication may suffer due to various reasons: The placement of nodes is not predetermined and external obstacles may cause poor communication performance between two nodes. If this type of problem is encountered then end to end communication will suffer. Another problem is failure of nodes due to battery depletion.

5. In sensor networks the loss of data, when it flows from source to sink is generally tolerable. But the data that flows from sink to source is sensitive to message loss. (A sensor obtains information from the surrounding environment and passes it on to the sink which in turn queries the sensor node for information)

Traditional transport protocols such as UDP and TCP cannot be directly implemented in sensor networks for the following reasons:

1. If a sensor node is far away from the sink then the flow and congestion control mechanism cannot be applied for those nodes.

2. Successful end to end transmissions of packets are guaranteed in TCP but it's not necessary in an event driven applications of sensor networks.

3. Overhead in a TCP connection does not work well for an event driven application of sensor networks. 
4. UDP on the other hand has a reputation of not providing reliable data delivery and has no congestion or flow control mechanisms which are needed for sensor networks.

Pump Slowly, Fetch Quickly (PSFQ) proposed in [32] one of the popular transport layer protocol.

Active Research Areas: A developing transport protocol for sensor networks is itself a difficult task due to the previously discussed issues and not much work is reported. Existing transport layer protocols for WSNs assume that the network layer uses a single path routing and multi path routing is not considered; which opens many doors for research in this direction. Many of the transport protocols do not consider priority when routing. Since sensor nodes are placed in various types of environment, the data from different locations will have different priorities.

3.3 Network layer issues: Over the past few years sensor networks are being built for specific applications and routing is important for sending the data from sensor nodes to Base Station (BS). As discussed in the introduction part, routing in sensor networks is a very challenging issue. Various issues at the network layer are [33, 34, and 35]:

1) Energy efficiency is a very important criterion. We need to discover different techniques to eliminate energy inefficiencies that may shorten the lifetime of the network. At the network layer, we need to find various methods for discovering energy efficient routes and for relaying the data from the sensor nodes to the BS so that the lifetime of a network can be optimized.

2) Routing Protocols should incorporate multi-path design technique. Multi-path is referred to those protocols which set up multiple paths so that a path among them can be used when the primary path fails.

3) Path repair is desired in routing protocols whenever a path break is detected. Fault tolerance is another desirable property for routing protocols. Routing protocols should be able to find a new path at the network layer even if some nodes fail or blocked due to some environmental interference.

4) Sensor networks collect information from the physical environment and are highly data centric. In the network layer in order to maximize energy savings we need to provide a flexible platform for performing routing and data management.

5) The data traffic that is generated will have significant redundancy among individual sensor nodes since multiple sensors may generate same data within the vicinity of a phenomenon. The routing protocol should exploit such redundancy to improve energy and bandwidth utilization.

6) As the nodes are scattered randomly resulting in an ad hoc routing infrastructure, a routing protocol should have the property of multiple wireless hops.

7) Routing Protocols should take care of heterogeneous nature of the nodes i.e. each node will be different in terms of computation, communication and power. Various type of routing Protocols for WSNs are Sensor Protocols for Information via negotiation (SPIN), Rumor Routing, Direct Diffusion, Low Energy Adaptive Cluster Hierarchy (LEACH), Threshold sensitive Energy Efficient sensor Network protocol (TEEN), Geographic and Energy Aware Routing (GEAR), Sequential Assignment Routing (SAR) and others

Active Research Areas: Sensor networks are still at an early stage in terms of technology as it is still not widely deployed in real world and this opens many doors for research. The current routing protocols need to be improved as they have their own set of problems. Much work is not reported on contention issues or high network traffic. Very little analytical work is done.

3.4 The data-link layer: The data link layer is concerned with the data transfer between two nodes that shares the same link. Since the underlying technology is wireless there is a need for energy efficient medium access control and management protocol. The protocol must put in mind, frame synchronization, fairness, bandwidth utilization, flow control and error control for data communication. Although several MAC protocols have been proposed the researcher may still take a look at system performance optimization, cross layer optimization, energy optimization and improving communication reliability.

3.5 The physical layer: The physical layer provides an interface for transmitting bit streams over the physical communication medium. The physical layer is responsible for interacting with the MAC layer, performing transmission and reception, and modulation. The physical layer must be designed with consideration of WSN requirements. Researchers must look into design of an energy efficient radio transmitter, optimal transmission power, good modulation scheme. Other WSN requirements that should be put into considerations at the physical layer design includes; Interference, synchronization and multi-casting. 


\section{System group issues}

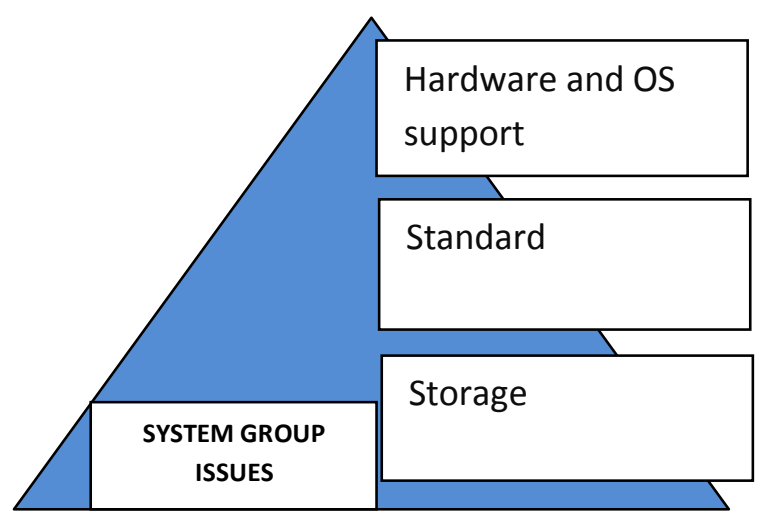

Fig 7: System Group Issues

For a sensor to operate in a WSN, there are several internal system issues that need to be addressed. This section takes a view of these issues.

4.1 Hardware and OS support: WSN platforms are built to support a wide range of sensor technology. Sensor products available in the markets have different radio components, processors, and storage. It is therefore a challenge to integrate different wireless sensors on a WSN platform since sensor hardware are different and processing raw data can be a problem with limited resources in the sensor node [1].System software such as the OS must be designed to support these sensor platforms. Research in this area involves designing platforms that support automatic management, optimizing network longevity, and distributed programming. [9] A Sensor is a device which senses the information and passes the same on to a mote. Sensors are used to measure the changes to physical environment like pressure, humidity, sound, vibration and changes to the health of person like blood pressure, stress and heartbeat. A Mote consists of processor, memory, battery, A/D converter for connecting to a sensor and a radio transmitter for forming an ad hoc network. A Mote and Sensor together form a Sensor Node. The structure of the sensor node is as shown in fig 3. There can be different Sensors for different purposes mounted on a Mote. Motes are also sometimes referred to as Smart Dust. A Sensor Node forms a basic unit of the sensor network [36, 37]. The nodes used in sensor networks are small and have significant energy constraints. The hardware design issues of sensor nodes are quite different from other applications and they are [38]:

1) Radio Range of nodes should be high (1-5 kilometres). Radio range is critical for ensuring network connectivity and data collection in a network as the environment being monitored may not have an installed infrastructure for communication. In many networks the nodes may not establish connection for many days or may go out of range after establishing connection.

2) Use of Memory Chips like flash memory is recommended for sensor networks as they are non-volatile, inexpensive and volatile.

3) Energy/Power Consumption of the sensing device should be minimized and sensor nodes should be energy efficient since their limited energy resource determines their lifetime. To conserve power the node should shut off the radio power supply when not in use. Battery type is important since it can affect the design of sensor nodes. Battery Protection Circuit to avoid overcharge or discharge problem can be added to the sensor nodes.

4) Sensor Networks consists of hundreds of thousands of nodes. It is preferred only if the node is cheap.

There are various platforms that are developed considering the above discussed design issues like Mica2, MicaZ, Telos, BT Node and Imotes and MIT $\mu$ AMPS ( $\mu$-Adaptive Multi-domain Power-aware Sensors) [39.40]. Among them the Berkeley Motes, which is commercially made available by Crossbow Technologies is very much popular and is used by various research organizations. Berkeley Motes consists of an embedded microcontroller, low-power radio, flash memory and they are powered by two AA batteries. MICA and MICA2 are the most successful families of Berkeley motes. The MICA2 platform is equipped with an Atmel ATmega128L and has a CC1000 transceiver. A 51-pin expansion connector is available to interface sensors. Microcontrollers are used to handle medium access and baseband processing. An event driven real time operating system like TinyOS has been implemented to specifically address the concurrency and resource management needs of sensor nodes [39, 40]. Fig 8 shows the Mica2 node. 


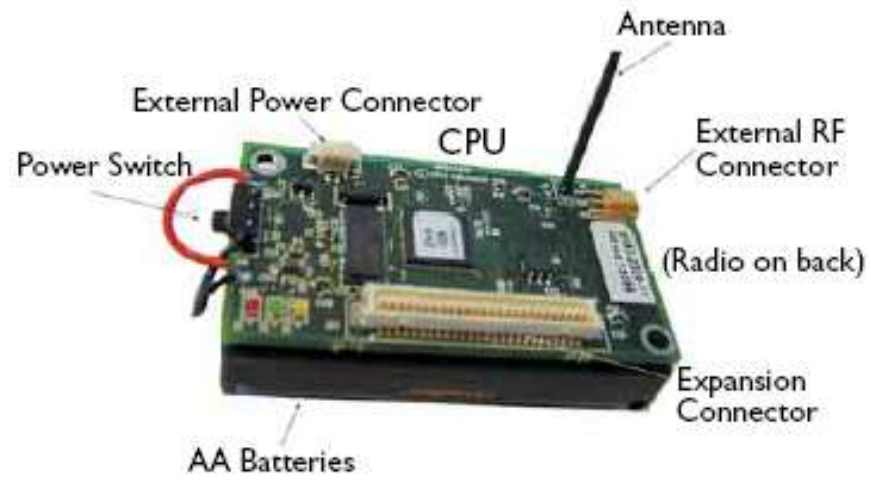

Fig 8. The Mica2 Sensor Node.

Active Research Areas: The research issues that can be considered are different strategies to improve signal reception, design of low power, less cost sensors and processing units. Various schemes to conserve node power consumption and node optimization and simple modulation schemes may also be considered for sensor nodes.

Operating system: An operating system framework for a sensor node should be able to provide memory management and resource management in a constrained environment. The various issues in designing an Operating System (OS) for sensor networks are:

1) In sensor network a sensor node is mainly responsible for computation of the extracted data from the local environment. It processes the extracted data and manipulates the data as per the requirement of an application. All these activities require real time response, processing and routing of the data. So concurrency management is needed in sensor nodes.

2) An OS for sensor nodes should be hardware independent and application specific. It should support multihop routing and simple user level networking abstractions.

3) The OS should have inbuilt features to reduce the consumption of battery energy. Motes cannot be recharged as and when wished due to small size and low cost requirement of motes and it should be in a position to enforce limitation on the amount of resources used by each application. The OS should be priority based and it should give precedence for higher priority events.

4) The OS should have an easy programming paradigm. Application developers should be able to concentrate on their application logic instead of being concerned with the low level hardware issues like scheduling, preempting and networking.

Various Operating Systems for Sensor nodes like TinyOS, Mantis Operating System [9] and Nano-Qplus [41] have been designed keeping in mind the above design issues. TinyOS is an open source and far by the most popular OS adapted by both the researchers and industry alike for embedded sensor networks. It has been ported on to many platforms and sensor devices. According to the information available on the TinyOS website [75] TinyOS has a component-based architecture enabling rapid innovation and implementation while minimizing the code size as required by the severe memory constraints which is inherent in sensor networks [76]. TinyOS's component library includes network protocols, distributed services, sensor drivers and data acquisition tools. The execution model of TinyOS supports complex yet safe concurrent operations. TinyOS has been implemented in NesC language [12], which supports the TinyOS component and concurrency model.

4.2 Standard: A standard specifies the functions and protocols necessary for sensor nodes to interface with a variety of networks. There are a number of standardization bodies in the field of WSNs. The IEEE focuses on the physical and MAC layers; the Internet Engineering Task Force works on layers 3 and above. In addition to these, bodies such as the International Society of Automation provide vertical solutions, covering all protocol layers. Some of the already established standards include the IEEE 802.15.4 [3], ZigBee [4, 5], Wireless HART [6, 7], ISA100.11 [8], IETF 6LoWPAN [9-11], IEEE 802.15.3 [12], Wibree [13]. Several standards are currently either ratified or under development for wireless sensor networks, standards in wireless networks must bear in mind WSNs unique characteristics mentioned in section one. [2]

4.3 Storage: Conventional approaches in WSNs require that data be transferred from sensor nodes to a centralized base station because storage is limited in sensor nodes. Techniques such as aggregation and compression reduce the amount of data transferred, thereby reducing communication and energy costs. These techniques are important for real-time or event-based applications, but they may not suffice. Applications that operate on a query-and collect approach will selectively decide which data are important to collect. Given that storage space is limited and communication is expensive, a storage model is necessary to satisfy storage constraints and query requirements. Though some storage models exist such as; Graph Embedding (GEM) [14], 
Two-tier sensor storage architecture (TSAR) [15] and Multi-resolution storage [16]. Emerging issues in storage models is anchored on the limited memory space and size as such the requirements for an efficient memory space include: (1) minimizing storage size to maximize coverage/data retention; (2) minimizing energy; (3) supporting efficient query execution on the stored data (note that in the reach back method where all the data must be sent to the observer, query execution is simply the transfer of the data to the observer)[ 17 ]; and (4) providing efficient data management under constrained storage. Several approaches to storage management have been proposed to meet the above requirements, with most approaches involving a trade off among these different goals. [9]

\section{Key management issues}

Before a WSN can exchange data securely, encryption keys must be established among sensor nodes. Key distribution refers to the distribution of multiple keys among the sensor nodes, which is typical in a nontrivial security scheme. Key management is a broader terms for key distribution, which also includes the processes of key setup, the initial distribution of keys, and key revocation - the removal of a compromised key. [42]

5.1 The need for key management: Key management, like security, is a cross-layered issue. The need for key management starts in the link layer. An applicable link layer standard in a WSN is IEEE 802.15.4. Although this standard considers key usage for secure data transmission, it does not specify how to exchange keys securely. This leaves open the key management problem that is the focus of much recent research. Besides the link layer, upper layers such as the network and application layers also must exchange keys securely. Many securitycritical applications depend on key management processes to operate but also demand a high level of fault tolerance when a node is compromised. This is a challenging problem because there are many stringent requirements for key management, and the resources available to implement such processes are highly constrained. [42]

5.2 Five key management protocols: Eschenauer, Du, LEAP, SHELL, and Panja. Eschenauer's scheme is a classical random key distribution scheme for WSNs. Du's scheme improves on Eschenauer's scheme by using key matrices. LEAP provides a highly flexible key management scheme using four types of keys. SHELL focuses on achieving high robustness, and Panja is optimized for hierarchical WSNs. LEAP, SHELL, and Panja support cluster-based operations and are more aligned with current trends as shown by the new standards, IEEE 802.15.4b and the ZigBee "enhanced" standard. Future developments likely will incorporate the features of LEAP and adjustable robustness enhancements from Eschenauer or Du; extremely security-critical applications may benefit from restructuring SHELL to ease implementation and maintenance. Developments for extremely large WSNs should consider improving Panja's scheme due to its hierarchical scalability feature. [42]

\section{Topology issues}

Topology issues have received more and more attentions in Wireless Sensor Networks (WSN). While WSN applications are normally optimized by the given underlying network topology, another trend is to optimize WSN by means of topology control. A number of approaches have been invested in this area, such as topology directed routing, cooperating schemes, sensor coverage based topology control and network connectivity based topology control. Most of the schemes have proven to be able to provide a better network monitoring and communication performance with prolonged system lifetime. [75] 


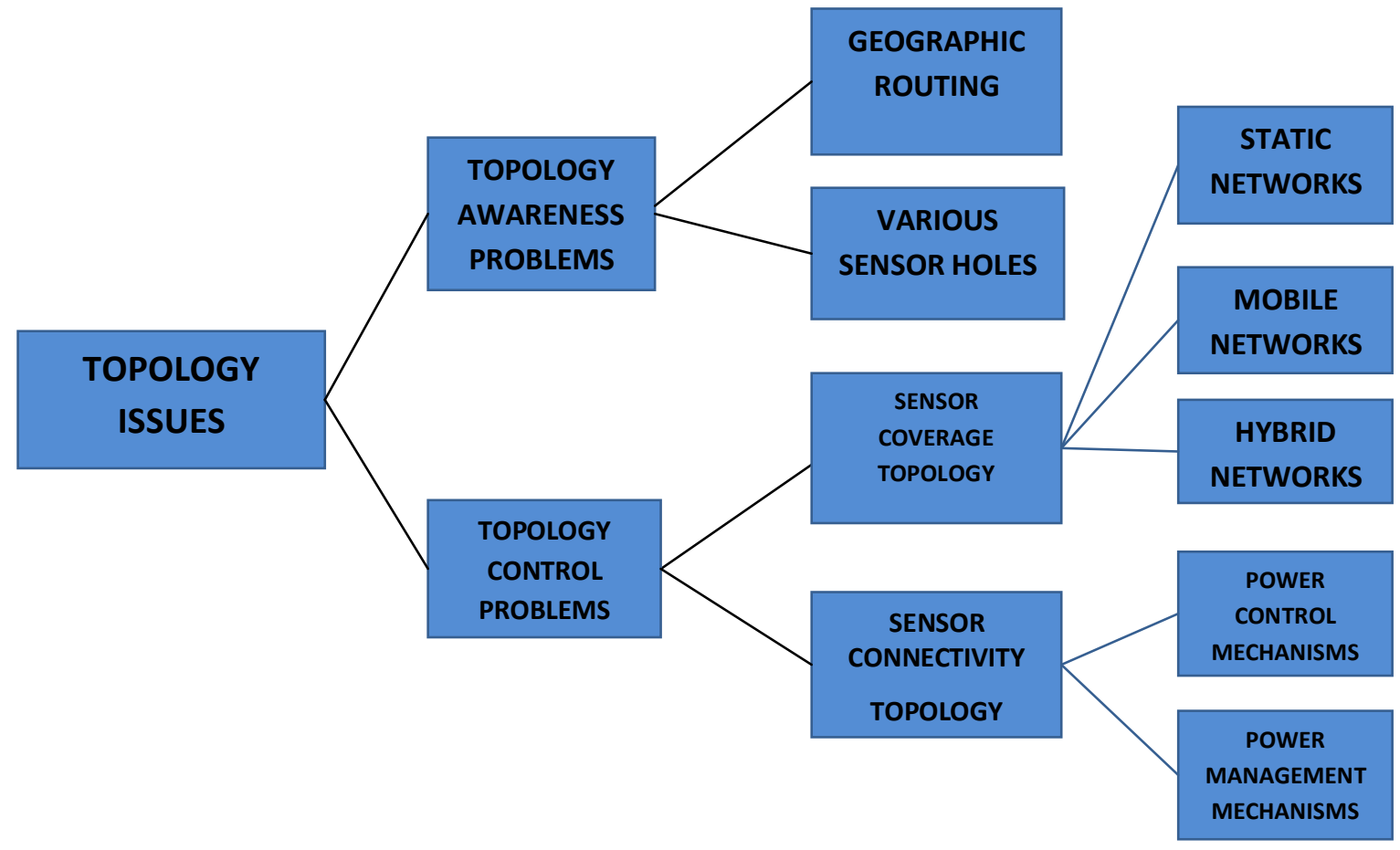

Fig 9: Taxonomy of topology issues in wsn Localization

X. Other wsn issues

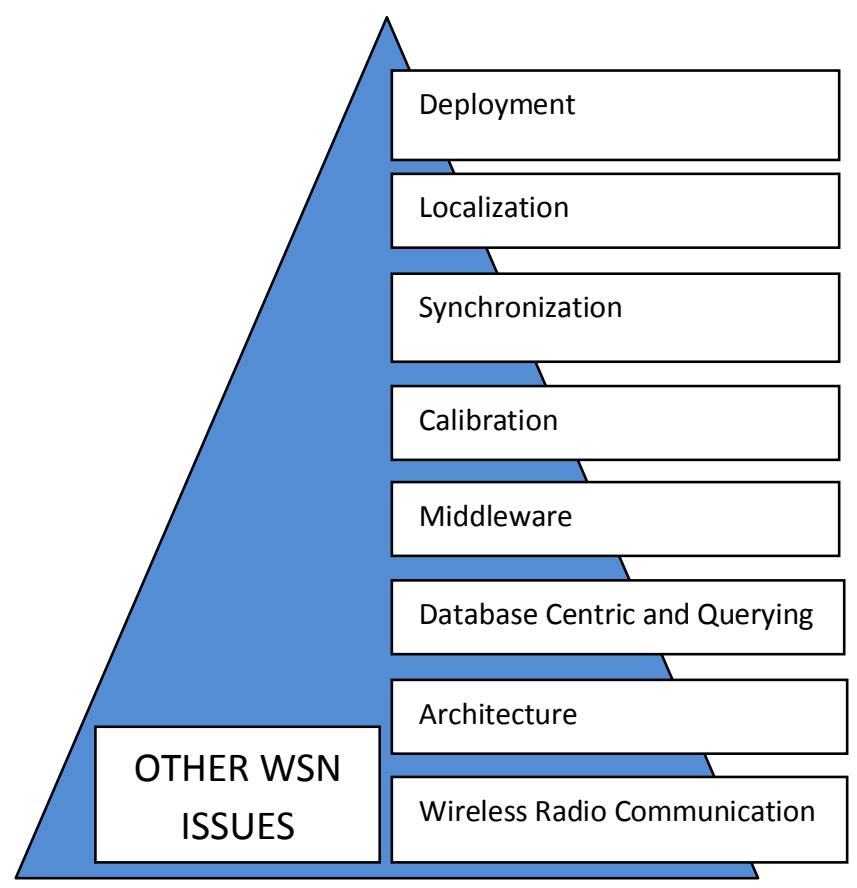

Fig 10: Other WSN Issues

7.1 Deployment: Deployment means setting up an operational sensor network in a real world environment [43]. Deployment of sensor network is a labor intensive and cumbersome activity as we do not have influence over the quality of wireless communication and also the real world puts strains on sensor nodes by interfering during communications. Sensor nodes can be deployed either by placing one after another in a sensor field or by dropping it from a plane. Various deployment issues which need to be taken care are [44, 45]: 
1) When sensor nodes are deployed in real world, Node death due to energy depletion either caused by normal battery discharge or due to short circuits is a common problem which may lead to wrong sensor readings. Also sink nodes acts as gateways and they store and forward the data collected. Hence, problems affecting sink nodes should be detected to minimize data loss.

2) Deployment of sensor networks results in network congestion due to many concurrent transmission attempts made by several sensor nodes. Concurrent transmission attempts occur due to inappropriate design of the MAC layer or by repeated network floods. Another issue is the physical length of a link. Two nodes may be very close to each other but still they may not be able to communicate due to physical interference in the real world while nodes which are far away may communicate with each other.

3) Low data yield is another common problem in real world deployment of sensor nodes. Low data yield means a network delivers insufficient amount of information.

4) Self Configuration of sensor networks without human intervention is needed due to random deployment of sensor nodes.

A framework is proposed in [45] considering the above deployment issues. POWER is a software environment for planning and deploying wireless sensor network applications into actual environment.

Active Research Areas: Research issues include improving the range and visibility of the radio antennas when deployed in various physical phenomenons, detecting wrong sensor readings at the earliest, to reduce latency and reduce congestion.

7. 2 localization: Sensor localization is a fundamental and crucial issue for network management and operation. In many of the real world scenarios, the sensors are deployed without knowing their positions in advance and also there is no supporting infrastructure available to locate and manage those once they are deployed [46, 47, 48].

Determining the physical location of the sensors after they have been deployed is known as the problem of localization. Location discovery or localization algorithm for a sensor network should satisfy the following requirements [49]:

1) The localization algorithm should be distributed since a centralized approach requires high computation at selective nodes to estimate the position of nodes in the whole environment. This increases signalling bandwidth and also puts extra load on nodes close to center node.

2) Knowledge of the node location can be used to implement energy efficient message routing protocols in sensor networks.

3) Localization algorithms should be robust enough to localize the failures and loss of nodes. It should be tolerant to error in physical measurements.

4) It is shown in [50] that the precision of the localization increases with the number of beacons. A beacon is a node which is aware of its location. But the main problem with increased beacons is that they are more expensive than other sensor nodes and once the unknown stationary nodes have been localized using beacon nodes then the beacons become useless.

5) Techniques that depend on measuring the ranging information from signal strength and time of arrival require specialized hardware that is typically not available on sensor nodes.

6) Localization algorithm should be accurate, scalable and support mobility of nodes.

Active Research Areas: The research on mobile nodes localization and motion analysis in real time will continue to grow as sensor networks are deployed in large numbers and as applications become varied. Scientists in numerous disciplines are interested in methods for tracking the movements and population counts of animals in their habitat i.e. passive habitat monitoring. Another important application is to design a system to track the location of valuable assets in an indoor environment. We need to improve the maximum likelihood estimation in a distributed environment like sensor networks. Developing mobile assisted localization is another important research area. One needs to improve the localization accuracy which depends on ToA or TDoA.

7.3 Synchronization: Clock synchronization is an important service in sensor networks. Time Synchronization in a sensor network aims to provide a common timescale for local clocks of nodes in the network. A global clock in a sensor system will help process and analyze the data correctly and predict future system behavior. Some applications that require global clock synchronization are environment monitoring, navigation guidance, vehicle tracking etc. A clock synchronization service for a sensor network has to meet challenges that are substantially different from those in infrastructure based networks [51.52, 53].

1) Energy utilization in some synchronization schemes is more due to energy hungry equipments like GPS (Global Positioning System) receivers or NTP (Network Time Protocol).

2) The lifetime or the duration for the nodes which are spread over a large geographical area needs to be taken into account. Sensor nodes have higher degree of failures. Thus the synchronization protocol needs to be more robust to failures and to communication delay. 
3) Sensor nodes need to coordinate and collaborate to achieve a complex sensing task like data fusion. In data fusion the data collected from different nodes are aggregated into a meaningful result. If the sensor nodes lack synchronization among themselves then the data estimation will be inaccurate.

4) Traditional synchronization protocols try to achieve the highest degree of accuracy. The higher the accuracy, then there will be more requirement for resources. Therefore we need to have trade off between synchronization accuracy and resource requirements based on the application.

5) Sensor networks span multi hops with higher jitter. So, the algorithm for sensor network clock synchronization needs to achieve multihop synchronization even in the presence of high jitter.

Various synchronization protocols which can be found in the literature are Reference Broadcast Synchronization (RBS) and Delay Measurement Time Synchronization protocol.

Active Research Areas: Various research issues include building analytical model for multihop synchronization, improving the radio communication in the existing synchronization protocols like RBS (Reference Broadcast Synchronization) and LTS (Lightweight Tree Based Synchronization).

7.4 Calibration: Calibration is the process of adjusting the raw sensor readings obtained from the sensors into corrected values by comparing it with some standard values. Manual calibration of sensors in a sensor network is a time consuming and difficult task due to failure of sensor nodes and random noise which makes manual calibration of sensors too expensive. Various Calibration issues in sensor networks are [54, 55, 56]:

1) A sensor network consists of large number of sensors typically with no calibration interface.

2) Access to individual sensors in the field can be limited.

3) Reference values might not be readily available.

4) Different applications require different calibration.

5) Requires calibration in a complex dynamic environment with many observables like aging, decaying, damage etc.

6) Other objectives of calibration include accuracy, resiliency against random errors, ability to be applied in various scenarios and to address a variety of error models.

Research includes designing various calibration techniques involving the various issues which we have discussed previously.

7.5 Middleware: A middleware for wireless sensor network should facilitate development, maintenance, deployment and execution of sensing-based applications. WSN middleware can be considered as a software infrastructure that glues together the network hardware, operating systems, network stacks and applications [57]. Various issues in designing a middleware for wireless sensor networks are [58, 59, 60, 61, 62, and 63]:

1) Middleware should provide an interface to the various types of hardware and networks supported by primitive operating system abstractions. Middleware should provide new programming paradigm to provide application specific API's rather than dealing with low level specifications.

2) Efficient middleware solutions should hide the complexity involved in configuring individual nodes based on their capabilities and hardware architecture.

3) Middleware should include mechanisms to provide real time services by dynamically adapting to the changes in the environment and providing consistent data. Middleware should be adaptable to the devices being programmed depending on the hardware capabilities and application needs.

4) There should be transparency in the middleware design. Middleware is designed for providing a general framework whereas sensor networks are themselves designed to be application specific. Therefore we need to have some tradeoff between generality and specificity.

5) Sensor network middleware should support mobility, scalability and dynamic network organization. Middleware design should incorporate real time priorities. Priority of a message should be assigned at runtime by the middleware and should be based on the context.

6) Middleware should support quality of service considering many constraints which are unique to sensor networks like energy, data, mobility and aggregation.

7) Security has become of paramount importance with sensor networks being deployed in mission critical areas like military, aviation and in medical field. Several middleware systems have been designed to deal with the aforementioned issues. Mate [64] is a middleware architecture for constructing application specific virtual machines that executes on top of TinyOS.

Active Research Areas: The design and implementation of a middleware layer for fully realizing the potential of wireless sensor network is an open research area which still needs to be investigated further. One needs to design developer friendly middleware architecture which is not only generic but also should take care of all the underlying hardware intricacies while helping to reduce the energy consumption and also provide adequate quality of support. 
7.6 Database centric and querying: Wireless sensor networks have the potential to span and monitor a large geographical area producing massive amount of data. So sensor networks should be able to accept the queries for data and respond with the results. The data flow in a sensor database is very different from the data flow of the traditional database due to the following design issues and requirements of a sensor network [65, 66, 67 and 68]:

1) The nodes are volatile since the nodes may get depleted and links between various nodes may go down at any point of time but data collection should be interrupted as little as possible.

2) Sensor data is exposed more errors than in a traditional database due to interference of signals and device noise.

3) Sensor networks produce data continuously in real time and on a large scale from the sensed phenomenon resulting in need of updating the data frequently; whereas a traditional database is mostly of static and centralized in nature.

4) Limited storage and scarce of energy is another important constraint that needs to be taken care of in a sensor network database but a traditional database usually consists of plenty of resources and disk space is not an issue.

5) The low level communication primitives in the sensor networks are designed in terms of named data rather than the node identifiers which are used in the traditional networks.

Active Research Areas: Some research areas in sensor database include providing Spatio-temporal querying, multiquery optimization, storage placement, designing a distributed long term networked data storage, should have low energy communication overhead, various ways of representing the sensor data, processing and distributing query fragments, dealing with communication failures and designing various models for deploying and managing a sensor database systems.

7.7 Architecture: According to the authors of [69] lack of an overall sensor network architecture is the main factor for currently limiting the progress in sensor networks. Architecture can be considered as a set of rules and regulation for implementing some functionality along with a set of interfaces, functional components, protocols and physical hardware. Software architecture is needed to bridge the gap between raw hardware capabilities and a complete system. The key issues that must be addressed by the sensor architecture are [70, 71, and 72]:

1) Several operations like continuous monitoring of the channel, encoding of data and transferring of bits to the radio need to be performed in parallel. Also sensor events and data calculations must continue to proceed while communication is in progress.

2) A durable and scalable architecture would allow dynamic changes to be made for the topology with minimum update messages being transmitted.

3) The system must be flexible to meet the wide range of target application scenarios since the wireless sensor networks to not have a fixed set of communication protocols that they must adhere to.

4) The architecture must provide precise control over radio transmission timing. This requirement is driven by the need for ultra-low power communication for data collection application scenarios.

5) The architecture must decouple the data path speed and the radio transmission rate because direct coupling between processing speed and communication bit rates can lead to sub-optimal energy performance. The authors of [73] design a novel SP abstraction which promotes cooperation across the link and network layers to utilize limited resources efficiently. A unifying abstraction in SP leads to supporting a variety of link-layer technologies and network protocols while taking care that doing so will not lead to a significant loss of efficiency.

7.8 Wireless radio communication characteristics: Performance of wireless sensor networks depends on the quality of wireless communication. But wireless communication in sensor networks is known for its unpredictable nature. Main design issues for communication in WSNs are:

1) Low power consumption in sensor networks is needed to enable long operating lifetime by facilitating low duty cycle operation, local signal processing.

2) Distributed Sensing effectively acts against various environmental obstacles and care should be taken that the signal strength, consequently the effective radio range is not reduced by various factors like reflection, scattering and dispersions.

3) Multihop networking may be adapted among sensor nodes to reduce communication link range and also density of sensor nodes should be high.

4) Long range communication is typically point to point and requires high transmission power, with the danger of being eavesdropped. So we should consider short range transmission to minimize the possibility of being eavesdropped.

5) Communication systems should include error control subsystems to detect errors and to correct them.

Active Research Areas: Research areas include designing low power consuming communication systems and complementary metal oxide semiconductor (CMOS) circuit technique specifically optimized for sensor 
networks, designing new architecture for integrated wireless sensor systems and modulation method and data rate selection. [9]

\section{Recent and research issues}

8.1 Production costs: Since the sensor networks consist of a large number of sensor nodes, the cost of a single node is very important to justify the overall cost of the networks. If the cost of the network is more expensive than deploying traditional single sensor devices, then the sensor network will not be cost justified. As a result, the cost of each sensor node has to be kept low. The cost for Bluetooth is usually less than $\$ 10$.The cost of a sensor node should be less than $\$ 1$ in order for sensor networks to be practically feasible .Current prices for sensor devices are much higher than even for Bluetooth. Furthermore, a sensor node may also have some additional units, e.g., for sensing and processing as described in Section 3.1. Also it may be equipped with a location finding system, mobilizer, or power generator depending on the applications of the sensor networks. These units all add to the cost of the sensor devices. As a result, the cost of a sensor node is a very challenging issue given the number of functionalities. [10]

8.2 Medical application issues: The emergence of wireless sensor networks (WSNs) in healthcare applications is gaining momentum through the increasing array of wearable vital sign sensors and location tags which can track healthcare personnel and patient status/ location continuously in real-time mode. Despite the increased range of potential application frameworks - ranging from pre-hospital, in-hospital, ambulatory and home monitoring, to long-term database collection for longitudinal trend analysis the security gap between existing WSN designs and the requirements of medical applications remains unresolved.[74]

8.3 Research issues: The severe constraints and demanding deployment environments of wireless sensor networks make computer security for these systems more challenging than for conventional networks. However, several properties of sensor networks may help address the challenge of building secure networks. First, we have the opportunity to architect security solutions into these systems from the outset, since they are still in their early design and research stages. Second, many applications are likely to involve the deployment of sensor networks under a single administrative domain, simplifying the threat model. Third, it may be possible to exploit redundancy, scale, and the physical characteristics of the environment in the solutions. If we build sensor networks so they continue operating even if some fraction of their sensors is compromised, we have an opportunity to use redundant sensors to resist further attack. Ultimately, the unique aspects of sensor networks may allow novel defenses not available in conventional networks. Many other problems also need further research. One is how to secure wireless communication links against eavesdropping, tampering, traffic analysis, and denial of service. Others involve resource constraints. Ongoing directions include asymmetric protocols where most of the computational burden falls on the base station and on public-key cryptosystems efficient on low end devices. Finally, finding ways to tolerate the lack of physical security, perhaps through redundancy or knowledge about the physical environment, will remain a continuing overall challenge. We are optimistic that much progress will be made on all of them.

\section{CONCLUSION}

The implementations of sensor networks in different areas have countless challenges but due to their versatile nature of applications, they have become more and more exciting for researchers as well as industry. They will prove to be a boon for next generation technology with the proper communication protocols developed. In the future, this wide range of application areas will make sensor networks an integral part of our lives. However there are certain issues discussed above like technical, design, topology, communication protocol, key management, research and recent and other issues should be taken care of to enhance the potential and the performance of sensor networks. These will help to enhance the applicability of wireless networks in various fields. Wireless sensor networks have become part of our networked world and their importance will tremendously grow and the future internet bridging the gap between digital and physical world.Context, ambient intelligence, and service creation platforms will play a key role. Actuation, in addition to sensing, is becoming more and more important. "Traditional" solutions developed in the past few years have not adequately addressed this new reality. Many technical issues are still to be fully addressed and solved. Successful approaches require advanced paradigms, interdisciplinary expertise, and cross-layer solutions. Despite the large amount of existing work, WSNs remain an exciting and open field of research.

\section{REFERENCES}

[1] "Henry Ohiani Ohize, Emerging Issues in Wireless Sensor Networks, Ohize, 2011

[2] H. Karl and A. Willig, Protocols and Architectures for Wireless Sensor Networks, John Wiley and Sons Ltd, The Atrium, Southern Gate, Chichester, West Sussex, England, 2005.

[3] D. Culler, D. Estrin, and M. Srivastava, "Overview of Sensor Networks", IEEE Computer, August 2004. 
[4] K. Martinez, J. K. Hart, and R. Ong, "Environmental sensor networks", IEEE Computer Journal, Vol. 37 (8), $50-56$, August 2004.

[5] A. Mainwaring, D. Culler, J. Polastre, R. Szewczyk , and J. Anderson, "Wireless sensor networks for habitat monitoring", Proceedings of the 1st ACM International workshop on Wireless sensor networks and applications, Atlanta, Georgia, USA, 88-97, 2002.

[6] http://en.wikipedia.org/wiki/Sensor_Networks

[7] Akyildiz, I.F., W. Su, Y. Sankarasubramaniam, E. Cayirci, "A Survey on Sensor Networks", IEEE Communications Magazine, August, 102-114(2002).

[8] Gowrishankar.S 1, T.G.Basavaraju 2, Manjaiah D.H 3, Subir Kumar Sarkar 4 "Issues in Wireless Sensor Networks", 2008

[9] Q. Jiang and D.Manivannan, "Routing Protocols for Sensor Networks", In proceedings of Consumer Communications and Networking Conference, 5-8 January, pp 93-98.

[10] Ian f.Alkyildiz and Mehmet Can Varun, Ian $\mathrm{f}$.Alkyildiz series in communications and networking, "wireless sensor networks book"

[11] "Configuration and tuning of sensor network applications through virtual sensors", Corsini, P. Dipt. di,Pisa Masci, P. ; Vecchio, A IEEE, March 2006

[12] "MAC \& Mobility In Wireless Sensor Networks", Marwan Al-Jemeli, Vooi Voon Yap and Fawnizu Azmadi Bin Hussin.

[13] "Security Issues in Wireless Sensor Networks Zoran S. Bojkovic, Bojan M. Bakmaz, and Miodrag R. Bakmaz, 2008.

[14] S. Al-Omari and W. Shi. Availability modeling and analysis of autonomous in-door wsns, Proceedings of IEEE MASS'07, September 2007

[15] B. Hamdaoui and P. Ramanathan. Energy-Efficient and MAC-Aware Routing for Data Aggregation in Sensor Networks, IEEE Press, October 2004

[16] C. Schurgers and M.B. Srivastava. "Energy efficient routing in wireless sensor networks", MILCOM Proceedings on Communications for Network-Centric Operations: Creating the Information Force, 2001.

[17] K. Seada, M. Zuniga, A. Helmy, and B. Krishnamachari. "Energy-efficient forwarding strategies for geographic routing in lossy wireless sensor networks", Proc. of ACMSenSys 2004, November 2004.

[18] R. Shah and J. Rabaey. "Energy aware routing for low energy ad hoc sensor networks",Proceedings of the IEEE Wireless Communications and Networking Conference(WCNC'02), March 2002.

[19] W. Ye, J. Heidemann, and D. Estrin. An energy-efficient mac protocol for wirelesssensor networks. In Proceedings of IEEE Infocom'02, New York, NY, June 2002.

[20] M. Younis, M. Youssef, and K. Arisha. "Energy-aware routing in cluster-based sensor network", Proceedings of ACM/IEEE MASCOTS'2002, October 2002.

[21] S. Jayashree, B. S. Manoj, and C. Siva Ram Murthy. On using battery state for medium access control in ad hoc wireless networks.In MobiCom '04: Proceedingsof the 10th annual international conference on Mobile computing and networking, pages 360-373, New York, NY, USA, 2004. ACM Press

[22] C. Schurgers et al. Topology management for sensor networks: exploiting latency and density. In Proceedings of the MobiHoc'02, June 2002

[23] Mark Yarvis, Nandakishore Kushalnagar, Harkirat Singh, Anand Rangarajan, York Liu, Suresh Singh, "Exploiting Heterogeneity in Sensor Networks".

[24] "QoS Support in Wireless Sensor Networks: A Survey", Dazhi Chen and Pramod K. Varshney.

[25] "Network connectivity in Wireless Sensor Networks:a Survey", Adnan Sultan, Madjid Merabti, Bob Askwith, Kashif KifayatSchool of Computing and Mathematical Sciences Liverpool John Moores University, (C) 2009

[26] "Some Target Coverage Issues of Wireless Sensor Network", Purnima Khuntia, Prasant Kumar Pattnaik, 2011

[27] Ramesh Rajagopalan and Pramod K. Varshney,"Data Aggregation Techniques in Sensor Networks: A Survey", IEEE Communications Surveys and Tutorials, 4th quarter, 2006.

[28] A.Boulis, Saurabh Ganeriwal, Mani B.Srivastava,"Aggregation in Sensor Networks: An energy-accuracy trade-off', Elseiver, Ad Hoc Networks 1 (2003) 317-331.

[29] "Wireless Sensor Network:Quality of Service (QoS) Issues and Challenges” Noor Zaman, Azween Abdullah Khalid Ragab, (C) 2012.

[30] C. Wang et.al, “A Survey of Transport Protocols for Wireless Sensor Networks”, IEEE Network June 2006, Vol: 20, Issue:3, pp:3440.

[31] V.S.Mansouri, B.Afsari, “A Simple Transport Protocol for Wireless Sensor Networks”, In proceedings of ISSNIP 2005

[32] Chien-Yih Wan, L.Krishnamurthy, "Pump-Slowly, Fetch-Quicklym (PSFQ): A Reliable Transport Protocol for Sensor Networks", IEEE Journal on selected areas in Communications, Vol 23, No 4, April 2005.

[33] Limin Wang,"Survey on Sensor Networks", Department of Computer Science Engineering, Michigan State University 2004

[34] Deepak Ganesan et.al, "Networking Issues in Wireless Sensor Networks", Elsevier Science, 9th December 2005.

[35] P.Jiang, Yu Wen et.al,"A Study of Routing Protocols in Wireless Sensor Networks", In Proceedings of the 6th World Congress on Intelligent Control and Automation, June 21-23, 2006, Dalian, China.

[36] M.Vieira, et.al., "Survey on Wireless sensor Network Devices", In proceedings of Emerging Technologies and Factory Automation, 2003 IEEE Conference, Volume: 1, 16-19, September 2003, pp: 537-544.

[37] http://computer.howstuffworks.com/mote1.htm

[38] P.Zhang, M.Sadler,A,Lyon and M.Martonosi, "Hardware Design Experiences in ZebraNet”, In proceedings of SenSys'04, November 3-5, 2004, Baltimore, USA.

[39] A.Sinha and A.Chandrakasan, "Dynamic Voltage Scheduling using adaptive filtering of workload traces", In proceedings of the $11^{\text {th }}$ International Conference on VLSI Design, 2001.

[40] A.P.Chandrakasan et.al, "An Architecure for a Power-Aware Distributed Microsensor Nodes", In IEEE workshop on Signal Processing Systems (SiPS’00), Lafayette, LA, Oct.2000.

[41] D.Puccinelli and M.Haenggi,"Wireless Sensor Networks: Applications and Challenges of Ubiquitous Sensing”, IEEE Circuits and Systems Magazine, Volume 5, Issue 3, 2005, pp: 19:31.

[42] Tahira Laskar, Debasish Jena, “A Survey on Key Management Issues in WSN”, 2012

[43] Matthias Ringwald, Kay Romer, "Deployment of Sensor Networks: Problems and Passive Inspection", In proceedings of Fifth International workshop on Intelligent solutions in embedded systems, Madrid, Spain 2007.

[44] Ashar Ahmed et.al, "Wired Vs Wireless Deployment Support for Wireless sensor Networks", TENCON 2006, IEEE region 10 conference, pp 1-3.

[45] J.Li, Y Bai, Haixing Ji and D. Qian, "POWER: Planning and Deployment Platform for Wireless Sensor Networks", In proceedings of the Fifth International Conference on Grid and Cooperative Computing Workshops (GCCW'06), IEEE 2006. 
[46] Xia Zhenjie, Chen Changjia,"A Localization Scheme with Mobile Beacon for Wireless Sensor Networks", In Proceedings of International Conference on ITS Telecommunications Proceedings 2006.

[47] S. Pandy, P Prasad, P.Sinha and P. Agarwal,'Localization of Sensor Networks considering energy accuracy tradeoffs", In proceedings of International conference on Collaborative computing: Networking, Applications and worksharing, 19-21 Dec 2005.

[48] Lingxuan Hu and David Evans,"Localization for mobile sensornetworks", In proceedings of the Tenth Annual International Conference on Mobile computing and Networking, Philadelphia 26th September-1 October 2004

[49] Ranjeet Kumar Patro,"Localization in Wireless Sensor Networks with mobile beacons", 23rd IEEE Convention of Electrical and Electronics Engineers in Israel, 2004, pp 22-24.

[50] A.Savvides, C.C.Han and M.B.Srivastava, "Dynamic fine grained Localization in Ad hoc networks of sensors", In proc of Mobicom 2001, pp 166-179, July 2001.

[51] Jeremy Elson, Kay Romer,"Wireless Sensor Networks: A New Regime for Time Synchronization", In proceedings of the First Workshop on Hop Topics in Networks 28-29 October 2002, Princeton, New Jersey, USA [52] Santashil PalChaudhuri, Amit Kumar Saha and David B.Johnson,"Adaptive Clock Synchronization in Sensor Networks", IPSN'04, April 26-27, Berkeley, California, USA.

[53] Fikret Sivrikaya and Bulent Yener,"Time Synchronization in Sensor Networks: A Survey", IEEE Network July/August 2004.

[54] Bychkovskiy V, Megerian S, Estrin D, and Potknojak M,"A Collaborative Approach to In place Sensor Calibration", 2nd International Workshop on Information Processing in Sensor Networks (IPSN'03), Polo Alto, April 2003, pages 301-316.

[55] J .Feng,S.Megerian and M.Potkonjak,"Model Based Calibration for Sensor Networks", Proceedings of IEEE Sensors, 22-24 october 2003, Vol: 2, pp:737-742.

[56] Kamin Whitehouse and David Culler,"Calibration as ParameterEstimation in Sensor Networks",In Proceedings of WSNA'02 September 28, 2002, Atlanta,Georgia,USA, pp 59-67.

[57] Salem Hadim, Nader Mohamed,"Middleware: Middleware Challenges and Approaches for Wireless Sensor Networks", IEEE Distributed Systems online 1541-4922, Published by the IEEE Computer Society, Vol 7, No 3, March 2006.

[58] Karen Henricksen and Ricky Robinson,"A survey of Middleware for Sensor Networks:State of the art and future directions", Proceedings of MidSens'06, November 27-December 1, 2006, Melbourne, Australia.

[59] M.Wolenetz,'Middleware Guidelines for Future Sensor Networks", First Annual International Conference on Broadband Networks, October 25-29, 2004, San Jose, California, USA

[60] Kay Romer, Oliver Kasten and F.Mattern,"Middleware Challenges for Wireless Sensor Networks", Mobile Computing and Communications Review, Volume 6, Number 2, Proceedings of the World Congress on Engineering 2008 Vol I WCE 2008, July 2 - 4, 2008, London, U.K. ISBN:978-988-98671-9-5 WCE 2008

[61] Yang Yu, Bhaskar Krishnamachari and Viktor K.Prasanna, "Issues in Designing Middleware for Wireless Sensor Networks", IEEE Network, January/February, IEEE 2004.

[62] Salem Hadim and Nader Mohammed,"Middleware for Wireless Sensor Networks", International conference on Parallel Processing Workshops, 2006, 14-18 August.

[63] M.M.Molla and Sheikh Iqbal Ahmad,"A survey of Middleware for Sensor Network and challenges", Proceedings of the 2006 International Conference on Parallel Processing Workshops (ICPPW'06), IEEE 2006

[64] P.Levis and D.Culler,"Mate: A Tiny Virtual Machine for Sensor Networks", In Proceedings of the 10th International Conference on Architectural Support for Programming Languages and Operating Systems, San Jose, CA, USA, October 2002.

[65] Li Qin Zhuang, Jing Bing Zhang, D.H.Zhang and Yi Zhi Zhao,"Data management for Wireless Sensor Networks:Research Issues and Challenges", In proceedings of 2005 International Conference on Control and Automation (ICCA2005), June 27-29, Budapest, Hungary.

[66] Philippe Bonnet, Johannes Gehrke and Praveen Seshadri,"Querying the physical world”, IEEE Personal Communications, October 2000.

[67] Julio C.Navas, Michael Wnyblatt,"The Network is the Database:Data Management for Highly Distributed Systems", ACM SIGMOD 2001 May 21-24, Santa Barbara, California USA.

[68] Amol Deshpande, C.Guestrin et.al,"Model-Driven Data Acquisition in Sensor Networks", Proceedings of the 30th VLDB Conference, Toronto, Canada, 2004.

[69] David Culler et.al,'Towards a sensor network architecture:Lowering the waistline",Proceedings of the hot topics of in Operating Systems (HotOS'05), International Computer Science Institute 2005.

[70] System Architecture for Wireless Sensor Networks by Jason Lester Hill, Ph.D dissertation, University of California at Berkeley.

[71] S.Duan and Xiaobu Yuan,'Exploring Hierarchy Architecture for Wireless Sensor Network Management”, IEEE 2006

[72] Jason Hill et.al,"System Architecture Directions for Networked Sensors", Proceedings of the ninth International conference on architectural support for programming languages and operating systems, pp: 93-104, November 2000, Cambridge, Massachusetts, USA.

[73] Joseph Polastre, Philip Levis, David Culler et.al,"A Unifying Link Abstraction for Wireless Sensor Networks", SenSys'05, November 2-4, 2005, San Diego, California, USA, ACM 2005.

[74] http://link.springer.com/article/10.1007\%2Fs10550-006-0051-8?LI=true.

[75] Mo Li, Baijian Yang, "A Survey on Topology issues in Wireless Sensor Network" 